Supporting information

\title{
Controlling Catalyst-Free Formation and Hole Gas Accumulation by Fabricating Si/Ge Core-Shell and Si/Ge/Si Core-Double Shell Nanowires
}

Xiaolong Zhang, ${ }^{\mathrm{a}, \mathrm{b}}$ Wipakorn Jevasuwan, ${ }^{\mathrm{a}}$ Yoshimasa Sugimoto, ${ }^{\mathrm{a}}$ and Naoki Fukata*a,b

anternational Center for Materials Nanoarchitectonics, National Institute for Materials

Science, Tsukuba, 3050044, Japan.

${ }^{\mathrm{b}}$ Graduate School of Pure and Applied Sciences, University of Tsukuba, Tsukuba, 3058573, Japan

*Email: FUKATA.Naoki@nims.go.jp

Vertically-aligned $p$-Si nanowires (NWs) with uniform diameters were formed by combining nanoimprint lithography, Bosch etching and chemical vapor deposition. Figure S1 shows the $p$-SiNW arrays before and after the thermal oxidation and wet etching process. The $\mathrm{O}_{2}$ annealing was carried out at $950{ }^{\circ} \mathrm{C}$, followed by three 10 -min cycles of HF etching. The diameter reduction caused by each cycle process could be detected, showing the reduction rate to be $50 \mathrm{~nm} / \mathrm{h}$, as shown in Figure $\mathrm{S} 1$ (e).
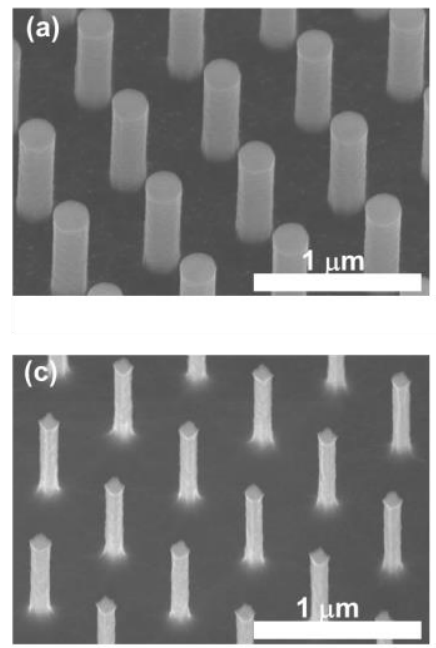
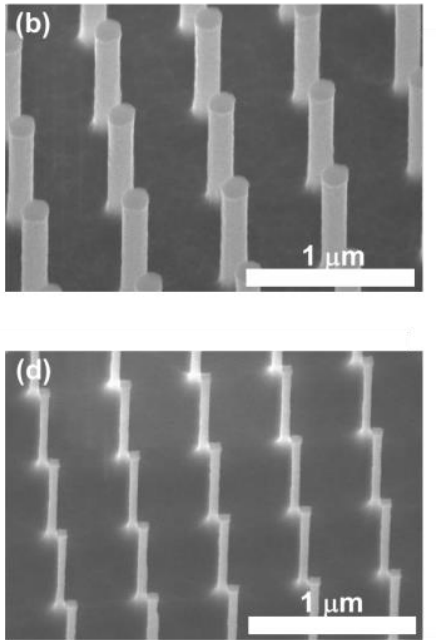

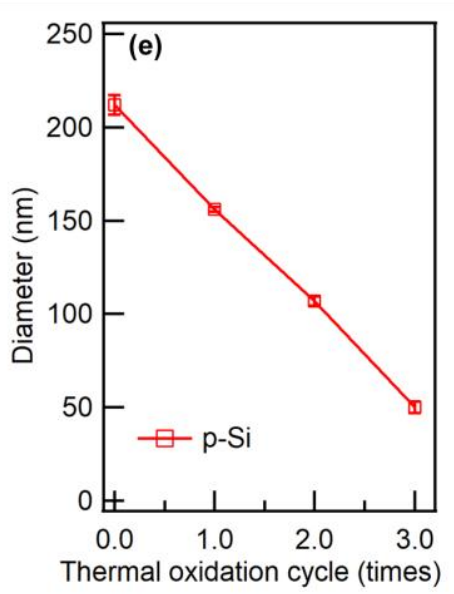

Figure S1. Thermal oxidation and wet etching process for reducing SiNW array diameter: (a) 0 cycles (as-grown), (b) 1 cycle, (c) 2 cycles, (d) 3 cycles, and (e) the diameter of SiNW array as a function of thermal oxidation and wet etching cycle time.

Figure S2 shows TEM images of a $p$-SiNW after three cycles of thermal oxidation and wet etching treatments. Figure S2 (a) is a low-resolution TEM image, demonstrating the maintained uniform NW structure. The high-resolution TEM images in Fig. S2 (b) show the singlecrystalline structure of the NWs. Thermal oxidation plus wet etching is therefore an effective 
strategy for controlling the NW diameter of a SiNW array.
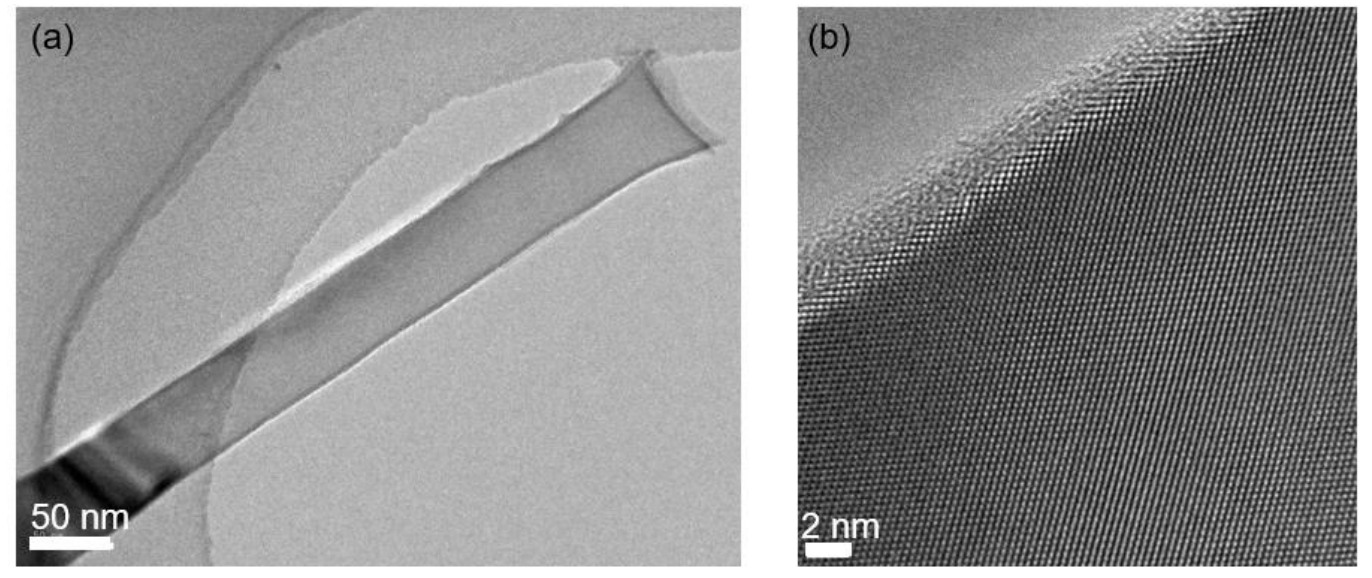

Figure S2. (a) Low- and (b) high-resolution TEM images after the thermal oxidation and wet etching process.

Figure S3 shows $p$-Si/intrinsic (i)-Ge core-shell NW arrays. The $i$-Ge shell layer was formed by epitaxial growth of Ge using CVD with a shell growth time of $80-160 \mathrm{~s}$, as shown in Figs. S3 (a) - (d). The total NW diameter was increased from $60 \mathrm{~nm}$ to $110 \mathrm{~nm}$, as shown in Figure S3 (e).
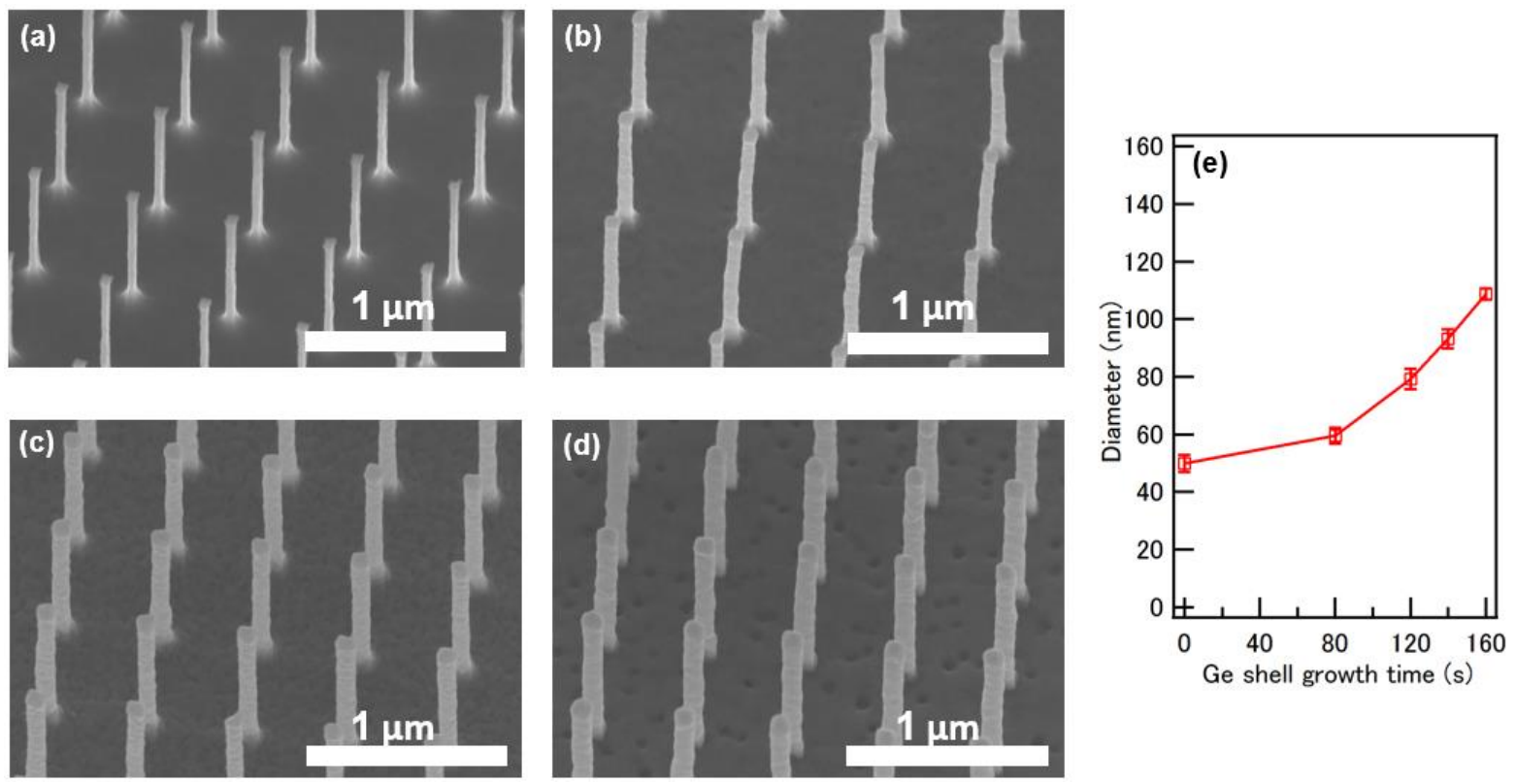

Figure S3. SEM images of $p$-Si $/ i$-Ge core-shell NW arrays formed using growth times of (a) $80 \mathrm{~s}$, (b) $120 \mathrm{~s}$, (c) $140 \mathrm{~s}$, (d) $160 \mathrm{~s}$; and (e) the diameter of the $p$-Si/i-Ge core-shell NW as a function of Ge shell growth time. 
X-ray diffraction (XRD) measurements were performed to investigate the strain induced in the core and shell regions. In Figure S4 (a), the Si (400) and Ge (400) crystallization peaks can be seen. The average lattice constants of the $p$-Si and $i$-Ge are plotted in Figs. S4 (b) and (c). The $i$-Ge lattice constant was reduced at shell growth times up to $120 \mathrm{sec}$, indicating an increase in compressive stress due to the thicker shell formation. The Ge lattice constant then increased up to the value obtained for bulk Ge. This is due to stress relaxation. The lattice constant of $p$-Si showed the opposite behavior. This is due to the increase in tensile stress induced from the $i$-Ge shell layer. The lattice constant of the $p$-Si finally approached the value of bulk Si due to stress relaxation.
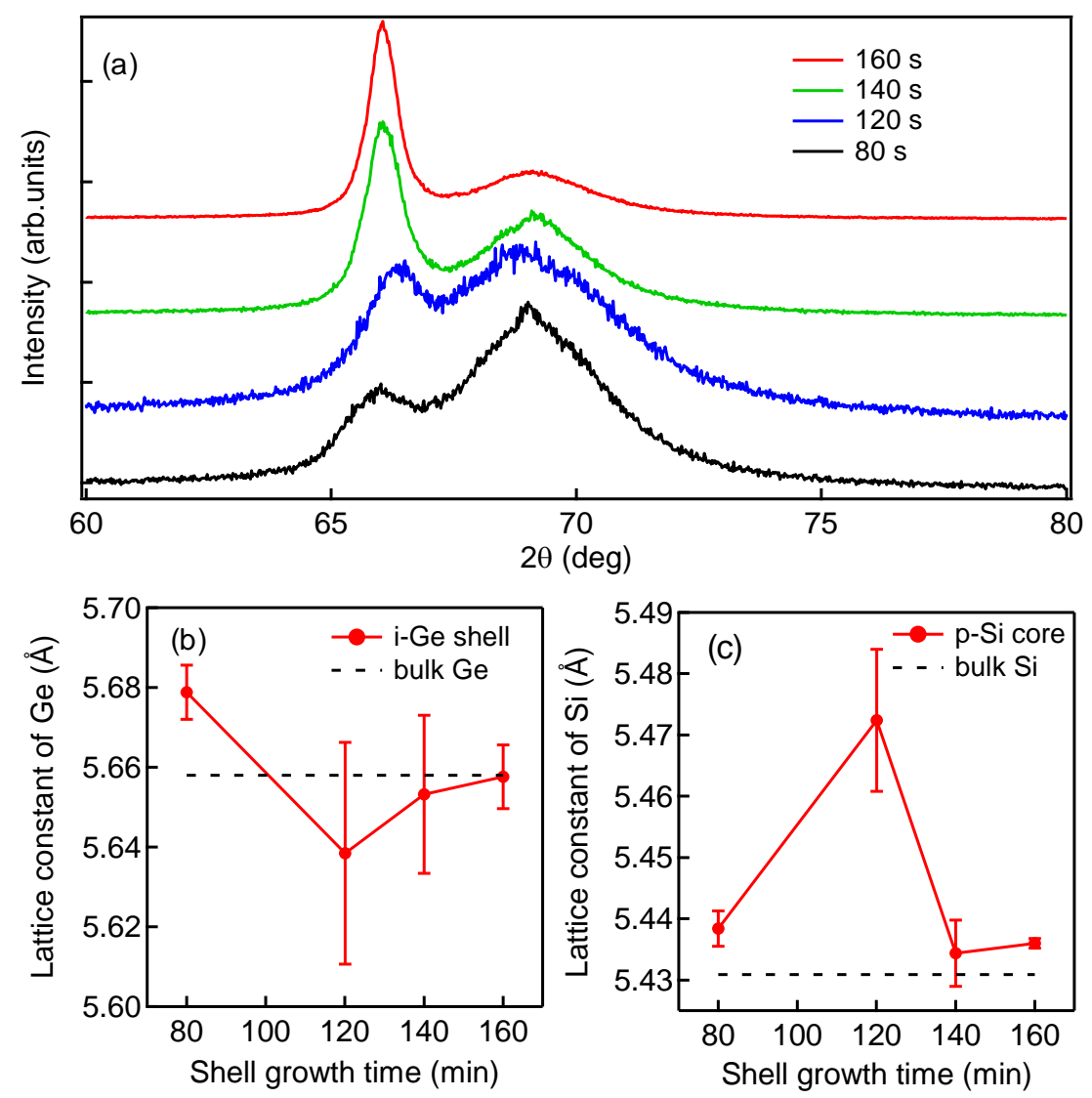

Figure S4. XRD spectra of $p$-Si/i-Ge core-shell NWs with various shell growth times from 80 - $160 \mathrm{~s}$; (a) Si (4 0 0) and Ge (4 0 0) peaks, (b) Ge, and (d) Si lattice constants as a function of shell growth time. The dashed lines are the lattice constants of bulk Ge and Si.

$p$-Si $/ i-\mathrm{Ge} / p$-Si NWs were fabricated under the fixed conditions of $50 \mathrm{~nm}$ for the $p$-Si core and $20 \mathrm{~nm}$ for the $i$-Ge shell, as shown in Figure S5. The $p$-Si shell layers were formed using shell growth times of $30 \mathrm{~s}, 60 \mathrm{~s}, 80 \mathrm{~s}$ and $100 \mathrm{~s}$. The resultant diameters of $p$-Si $/ i$-Ge $/ p$-Si coredouble shell NWs were about $95 \mathrm{~nm}, 130 \mathrm{~nm}, 140 \mathrm{~nm}$ and $155 \mathrm{~nm}$, as shown in Figure S5 (e). 

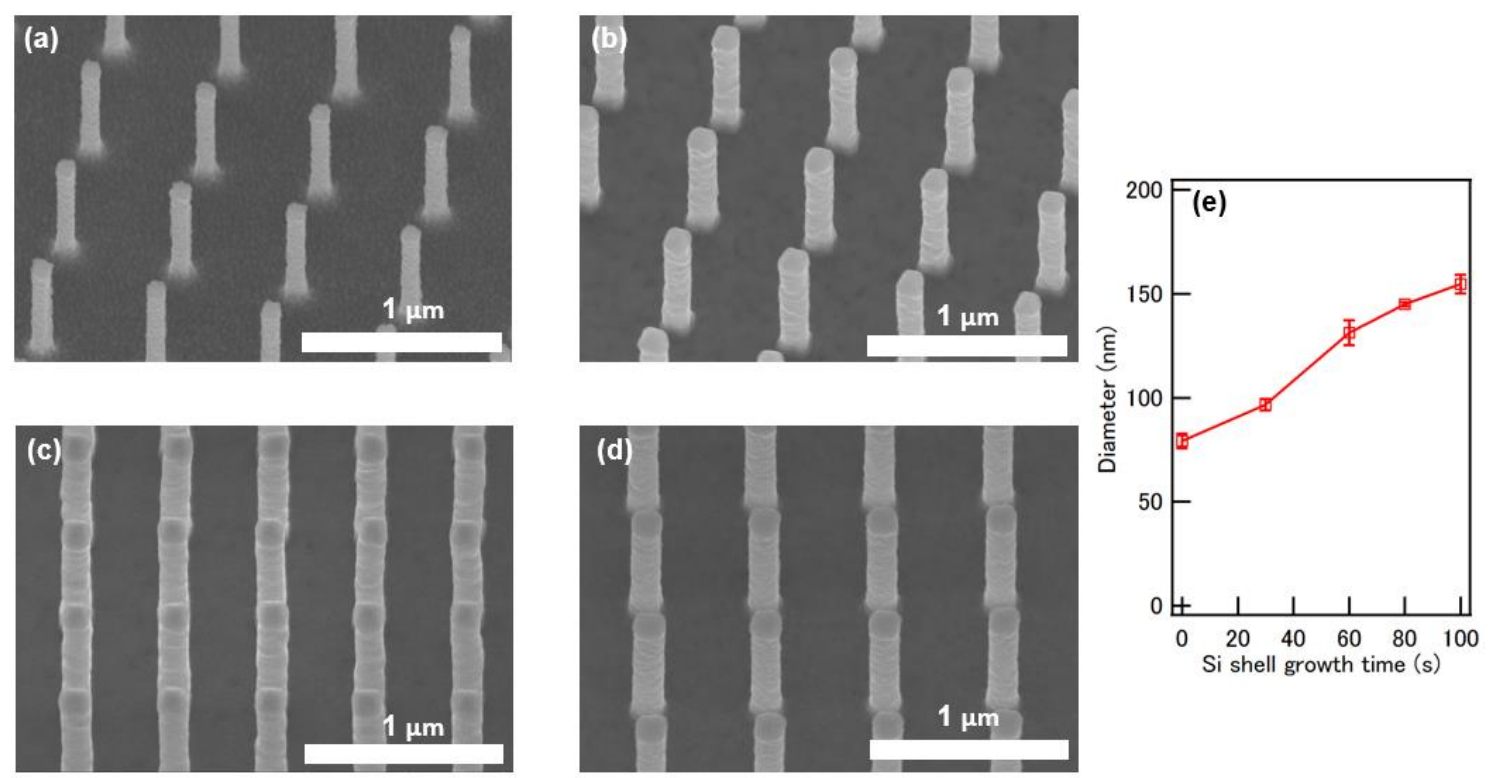

Figure S5. SEM images of $p$-Si $/ i$-Ge/ $p$-Si core-double shell NWs after applying flowing $\mathrm{SiH}_{4}(19 \mathrm{sccm})$ and $\mathrm{B}_{2} \mathrm{H}_{6}(0.5 \mathrm{sccm})$ gases with various times of (a) $30 \mathrm{~s}$, (b) $60 \mathrm{~s}$, (c) 80 $\mathrm{s}$, (d) $100 \mathrm{~s}$, and (e) the diameter of NW as a function of $p$-Si shell growth time. 